\title{
Miniaturization of Cavity-Backed Antenna Using Quarter Mode SIW Technique
}

\author{
Xiangchenyang Su${ }^{1,}$, Dongfang Guan ${ }^{2, b}$, Jihui Shi, ${ }^{1, c}$, Jianguo Zhang ${ }^{2, d}$ \\ ${ }^{1}$ Luoyang Electronic Equipment Test Center of China, Luoyang, China \\ ${ }^{2}$ College of Communications Engineering, PLA University of Science and Technology, Nanjing, \\ China \\ a ssxx729@hotmail.com, b gdfguandongfang@163.com, ${ }^{\mathrm{c}}$ jihuishi@hotmail.com, \\ dzhangjianguo@163.com
}

Keywords: antenna; cavity-back; miniaturization; quarter mod; substrate integrated waveguide.

\begin{abstract}
A novel substrate integrated waveguide (SIW) cavity-backed antenna using quarter mode SIW is proposed in this paper. The quarter mode SIW antenna is realized by splitting the SIW cavity into four parts along the fictitious quasi-magnetic wall, which can preserve the field distribution of original SIW. A leaky wave radiation can be obtained from the dielectric aperture of the fictitious quasi-magnetic wall. The proposed antenna not only has the advantages of conventional SIW cavity-backed antennas including low profile, light weight, high front-to back ratio, and easy integration with planar circuits, but also has the advantages of miniaturization and simple structure. To validate the concept, the antenna is fabricated and measured. The measurement results match with the simulation results very well.
\end{abstract}

\section{Introduction}

Substrate integrated waveguide (SIW) has been proposed to design many high-quality microwave and millimeter wave components because of its advantages of low profile, low insertion loss, low interference and easiness of integrating with planar circuits. Many types of SIW structure antennas and arrays have been extremely investigated in recent years, such as SIW slot array antennas, SIW cavity-backed antennas.

SIW cavity-backed antennas can be constructed completely at a single substrate including their backed cavities and feeding elements, which have high radiation performance and retain advantages of low-profile, low-cost fabrication [1]-[5]. Various techniques have been proposed to be applied in design of SIW cavity backed antennas, such as circularly polarization, bandwidth enhancement [1]-[3]. However, the size of SIW may be too large for some practical circuits. Realizing miniaturization is an important issue in design of SIW cavity backed antennas, especially in low frequency application. In [4], miniaturized SIW cavity-backed antennas using negative order resonance are proposed and developed. However, this technique is complex and cannot be used in all types of SIW cavity-backed antennas. An improved guided wave structure, called "half mode substrate integrated waveguide (HMSIW)" is proposed in [6]. SIW can be bisected along a fictitious quasi-magnetic wall and becomes an HMSIW, which can preserve the field distribution of the original SIW. Comparing with SIW, HMSIW not only has all superiorities, but also owns half size. The HMSIW has already been exploited to design some high-performance microwave components. For example, many SIW slot array antennas have used the HMSIW technique to realize miniaturization. The concept of HMSIW is first used in cavity-backed antenna in [5]. The results show that the proposed antennas have the radiation performance comparable to that of conventional metallic cavity backed antennas while only have the half size.

In this paper, in order to reduce the size of antenna further, we bisect the HMSIW cavity-backed into two parts again along the fictitious quasi-magnetic wall and a quarter mode SIW is realized. The quarter mode SIW can also preserve the field distribution of the original SIW and a leaky wave radiation can be obtained from the dielectric aperture of the fictitious quasi-magnetic wall. The quarter mode SIW antenna is proposed to reduce the size of SIW structure by nearly $75 \%$ without 
deteriorating its performance.

\section{Antenna Design}

The evolution process from original SIW to quarter mode SIW is shown in Fig. 1. A square SIW cavity is designed first. The SIW cavity uses a coaxial probe to feed at corner and is designed on Rogers RT5880 substrate with thickness of $1.57 \mathrm{~mm}$, low dielectric constant of 2.2. Through adjusting the size of SIW cavity, it is easy to make the resonant frequency of main mode $\mathrm{TE}_{110}$ work at $2.4 \mathrm{GHz}$. According to the theory of HMSIW, along the symmetrical plane of $\mathrm{TE}_{110}$ mode, the magnetic fields equal to zero and then this symmetrical plane can be considered as equivalent quasi-magnetic wall. SIW can be bisected along the fictitious quasi-magnetic wall and the SIW becomes an HMSIW. The HMSIW can preserve the field distribution of the original SIW.

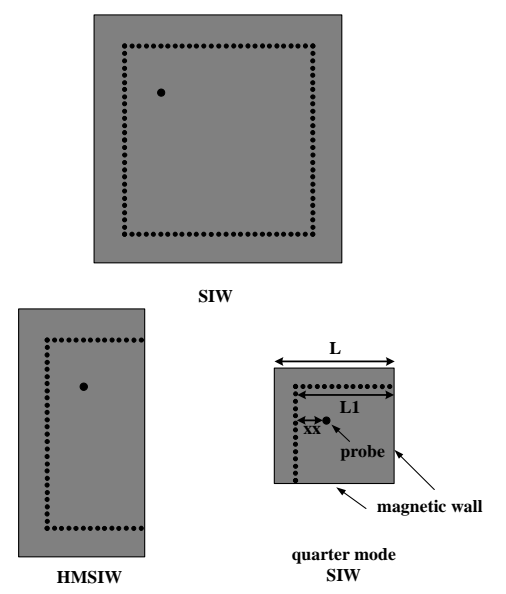

Figure 1. The evolution of the proposed quarter mode SIW antenna from SIW

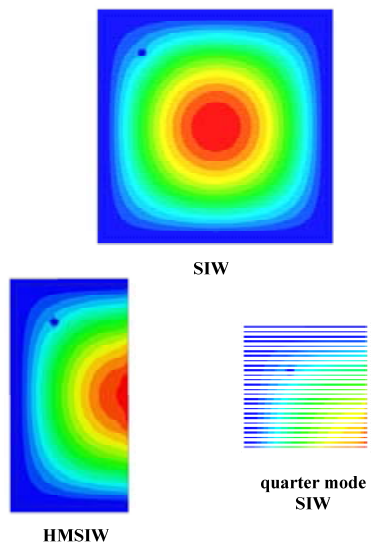

Figure 2. Electric field distribution of SIW, HMSIW, and quarter mode SIW

For square SIW cavity, there are two orthogonal equivalent quasi-magnetic walls. In order to reduce the size of antenna further, the HMSIW cavity-backed can be bisected into two parts again along the other fictitious quasi-magnetic wall and a quarter mode SIW is realized. The quarter mode SIW can also preserve the field distribution of the original SIW. As shown in Fig. 1, the quarter mode SIW consists of two metallic posts walls and two dielectric walls. The metallic posts can be considered as equivalent quasi-electric walls and the two dielectric walls can be considered as equivalent quasi-magnetic walls. Fig. 2 depicts the electric field distribution in three SIW cavities at 2.4 GHz. It is noted that the electric field distributions in HMSIW and quarter mode SIW are coincident with the distribution in original SIW. In quarter mode SIW, the maximum electric fields are along the equivalent quasi-magnetic walls. 


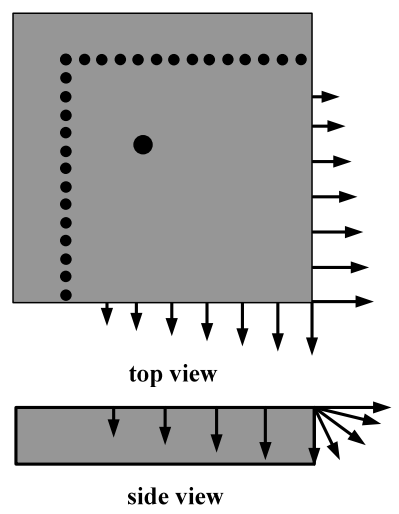

Figure 3. Generation of radiating electric field across the dielectric aperture

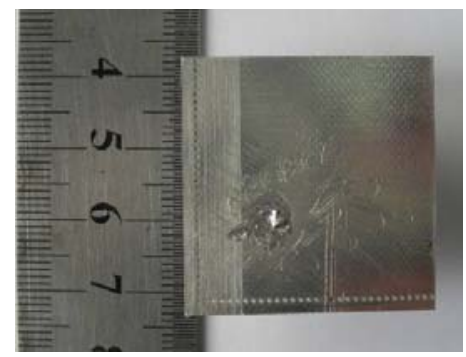

Figure 4. Photograph of the fabricated antenna

In antenna structure, there exists no radiating slot in quarter mode SIW cavity and the fields are radiated through the dielectric aperture. Because the fringing fields exist at the two magnetic walls, the open edges can act as radiators. In Fig. 3, it is illustrated how the power inside the cavity is radiated to free space through the dielectric aperture. This antenna radiates via its quasi-magnetic walls. In fact the radiation mechanism of this antenna is similar to that of the conventional microstrip antennas. In order to make the magnitudes of the two orthogonal electric fields equal, the feeding point must keep at the diagonal line of the cavity. Through tuning the feeding point, the energy can radiate effectively. Because the electric fields of two orthogonal equivalent quasi-magnetic walls have the same magnitude and phase, the radiating wave of the antenna is linearly polarized. The total polarized direction of electric fields is along the diagonal line of the cavity.

After optimization with Ansoft HFSS, the geometry parameters for the antenna are fixed as $\mathrm{L}=32 \mathrm{~mm}, \mathrm{~L} 1=30 \mathrm{~mm}, \mathrm{xx}=10.5 \mathrm{~mm}$. To validate the concept of the quarter mode SIW cavity-backed antenna and the research results above, the antenna is fabricated and measured. Photograph of the fabricated antenna is shown in Fig. 4.

\section{Results and Discussions}

Fig. 5 depicts simulated and measured return loss of the antenna. The measured results follow the trend of the simulated ones well with a slight frequency shifting which may be caused by the fabrication tolerance, material parameters inaccuracy, or measurement errors. The simulated return loss below $-10 \mathrm{~dB}$ is from $2.36 \mathrm{GHz}$ to $2.4 \mathrm{GHz}$, while the measured result is from $2.38 \mathrm{GHz}$ to 2.43 GHz. The measured impedance bandwidth reaches up to $2.1 \%$.

The simulated and measured normalized radiation patterns of the antenna in E-plane and H-plane at $2.41 \mathrm{GHz}$ are plotted in Fig. 6. The measured radiation patterns are in good agreement with the simulated ones in two orthogonal cut planes. Their $3 \mathrm{~dB}$ beamwidths in E-plane and $\mathrm{H}$-plane are about 1500 and 1600, respectively. The H-plane radiation patterns are symmetrical, but for the E-plane radiation patterns, the asymmetrical structure of the antenna makes the largest radiation direction offset from its boresight direction.

The measured peak gain is $1.66 \mathrm{dBi}$ at $2.41 \mathrm{GHz}$. In the range of measured impedance bandwidth from $2.38 \mathrm{GHz}$ to $2.43 \mathrm{GHz}$, the measured gain is above $1.25 \mathrm{dBi}$. Because the aperture size of the 
antenna reduces to a quarter comparing with original SIW antenna, the gain of the proposed antenna is not high.

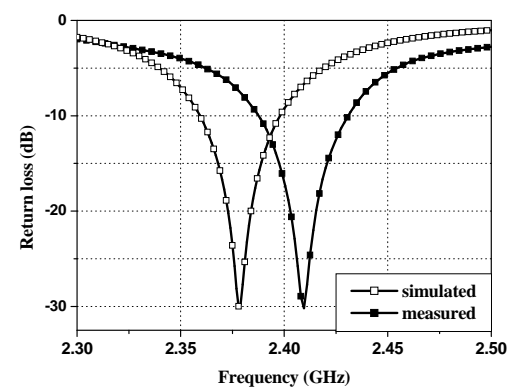

Figure 5. Simulated and measured return loss of the antenna

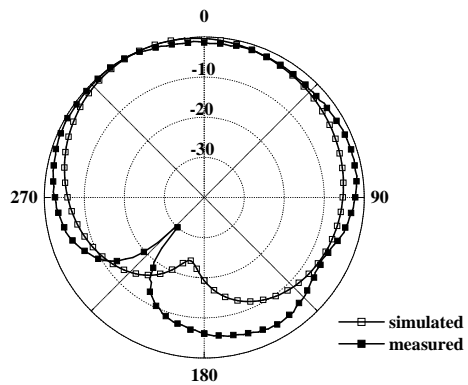

(a) E-plane

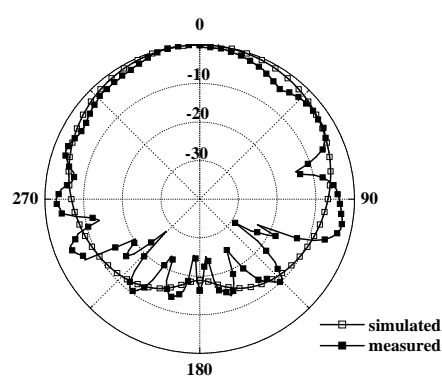

(b) H-plane

Figure 6. Radiation patterns of the proposed antenna at $2.41 \mathrm{GHz}$.

\section{Acknowledgements}

This work was supported in part by the National Science Foundation of China under Grant 61271103 and in part by the National Science Foundation of China under Grant 61271105.

\section{References}

[1] S. Yun, D. Y. Kim and S. Nam, "Bandwidth Enhancement of Cavity-Backed Slot Antenna Using a Via-Hole Above the Slot,” in IEEE Antennas Wireless Propag. Lett., vol. 11, pp. 1092-1095, 2012.

[2] G. Q. Luo, Z. F. Hu, Y. P. Liang, L. Y. Yu, and L. L. Sun, "Development of Low Profile Cavity Backed Crossed Slot Antennas for Planar Integration,” in IEEE Trans. Antennas Propag., vol. 57, No. 10, October 2009, pp. 2972-2978

[3] G. Q. Luo, Z. F. Hu, W. J. Li, X. H. Zhang, L. L. Sun, and J. F. Zheng, “Bandwidth-Enhanced Low-Profile Cavity-Backed Slot Antenna by Using Hybrid SIW Cavity Modes,” in IEEE Trans. Antennas Propag., vol. 60, No. 4, Apr. 2012, pp. 1698-1704.

[4] Yuandan Dong, and Tatsuo Itoh, "Miniaturized Substrate Integrated Waveguide Slot Antennas Based on Negative Order Resonance,” in IEEE Trans. Antennas Propag., vol. 58, No. 12, Dec. 2010, pp. 3856-3864.

[5] S. A. Razavi, and M. H. Neshati, "Development of a Linearly Polarized Cavity-Backed Antenna Using HMSIW Technique,” in IEEE Antennas Wireless Propag. Lett., vol. 11, pp. 1307-1310, 2012.

[6] W. Hong, B. Liu, Y. Q. Wang, Q. H Lai, and K. Wu, "Half mode substrate integrated waveguide: a new guide wave structure for microwave and millimeter wave application,” in Proc. Joint 31st Int. Conf. Terahertz Electron, Shanghai China, Sep. 2006. 